\title{
Nosocomial Burkholderia cepacia infections in a Turkish university hospital: a five-year surveillance
}

\author{
Murat Dizbay, Ozlem Guzel Tunccan, Busra Ergut Sezer, Firdevs Aktas, Dilek Arman \\ Department of Infectious Diseases and Clinical Microbiology, Gazi University School of Medicine, Ankara, Turkey
}

\begin{abstract}
Background: Burkholderia cepacia has the potential to cause fatal infections in ICUs, and multidrug resistance makes them a serious threat in hospital settings. The aim of this study was to evaluate the epidemiology of B. cepacia infections in our hospital.

Methodology: The incidence, clinical characteristics, antimicrobial susceptibility, and outcomes of nosocomial B. cepacia infections during a five-year period were retrospectively analysed according to the infection control committee records.

Results: A total of 39 cases with nosocomial B. cepacia infection were included in the study. B. cepacia was identified from $0.7 \%$ of the nosocomial isolates. Its incidence was 0.26 per 1,000 admissions with $53.8 \%$ crude mortality rate. The most frequent nosocomial $B$. cepacia infection was pneumonia (58.9\%), followed by bloodstream infections (25.6\%), surgical site infections (7.6\%), urinary tract infections, (5.1\%), and skin-soft tissue infections (2.5\%). Nosocomial B. cepacia infections were most commonly observed in intensive care units $(61.5 \%)$. The most active antimicrobial agents were piperacillin-tazobactam, cefoperazone-sulbactam, and carbapenems.

Conclusions: The incidence of nosocomial B. cepacia infections was rare in our hospital, and no outbreak was detected during the study period. However, infections caused by B. cepacia should be taken into consideration because of their high mortality due to multidrug resistance in ICU settings.
\end{abstract}

Key words: Nosocomial, Burkholderia cepacia, surveillance

J Infect Dev Ctries 2009; 3(4):273-277.

Received 8 January 2009 - Accepted 9 March 2009

Copyright $\odot 2009$ Dizbay et al. This is an open-access article distributed under the Creative Commons Attribution License, which permits unrestricted use, distribution, and reproduction in any medium, provided the original work is properly cited.

\section{Introduction}

Burkholderia cepacia, formerly Pseudomonas cepacia, is an aerobic, motile, glucosenonfermenting, multidrug resistant Gram-negative bacillus that proliferates under conditions of minimal nutrition and can survive in the presence of certain disinfectants. It is widely distributed in the natural environment and has been isolated from water, soil, fruits, and vegetables. B. cepacia has emerged as an serious human pathogen in the last two decades, causing fatal necrotizing pneumonia and bacteremia, especially in patients with cystic fibrosis $(\mathrm{CF})$ or chronic granulomatous disease [1-4]. B. cepacia was isolated in $0.2-0.6 \%$ of all ventilator-associated pneumonias reported to the National Nosocomial Infections Surveillance System (NNIS) in the United States between 1998 and 2004 [2,5]. B. cepacia has been associated with outbreaks involving infections of the bloodstream, respiratory tract, and urinary tract inintensive care unit (ICU) settings [4]. Early detection and treatment with appropriate antibiotics of this organism is important because of its high transmissibility in the hospital setting, intrinsic resistance to many antibiotics, and association with a poor prognosis. Though infrequent, $B$. cepacia infections can be severe, with reported mortality rates as high as $83 \%$ among patients with lower respiratory tract infections [6,7].

In this retrospective study, we analyzed the incidence, clinical characteristics, antimicrobial susceptibility, and outcomes of nosocomial $B$. cepacia infections during the period 2003 to 2007 in our institution.

\section{Materials and Methods}

Gazi University School of Medicine is a 1,000bed tertiary care teaching hospital in Ankara, Turkey. From January 2003 to December 2007, all patients with nosocomial infections (NI) due to B. cepacia were included in the study. The patients colonized with $B$. cepacia were excluded from the study. The data were obtained from infection control committee records. NI surveillance was performed actively, based on both laboratory and patient records, during 
the period. The diagnosis of NI was made according to Centers for Diseases Control and Prevention (CDC) criteria [8]. The following data of the patients were collected and analyzed: age, sex, wards, duration of hospitalization, presence of prior antibiotic treatment, risk factors and underlying diseases of the patients, site of NI, microbiological data, and outcome.

Microorganisms were identified by the BBL Crystal Enteric/Nonfermenter ID Kit (Becton Dickinson, USA). The in vitro activities of antimicrobial agents were tested against the clinical isolates of $B$. cepacia by a disk diffusion method according to the Clinical and Laboratory Standards Institute (CLSI) standards [9]. Mueller-Hinton agar plates were inoculated with a bacterial suspension equivalent to a 0.5 McFarland Standard and then antibiotic susceptibility disks (Oxoid) were applied. Zones of growth inhibition were recorded in milimeters after overnight incubation at $35^{\circ} \mathrm{C}$.

\section{Results}

During the study period, B. cepacia was isolated from various clinical specimens of 48 patients. Only 39 of them $(81.2 \%)$ were accepted as a causative agent of nosocomial infection. B. cepacia, as an aetiological agent, constituted $0.7 \%$ of NI isolates and $1.0 \%$ of Gram negative microorganisms. Nosocomial infection incidence was 30.5 per 1,000 admissions. Nosocomial $B$. cepacia infection incidence was 0.26 per 1,000 admissions. There was no acummulation of $B$. cepacia infections according to the occurrence time and the wards during the study period. The incidence of B. cepacia infections, its percentage among nosocomial pathogens, wards, and sites of NIs by years are shown in table 1 .

The male/female ratio was 1.29 with the mean age $54.4 \pm 23.4$ years (median 54 years). Mean duration of hospitalization was $15.2 \pm 9.9$ days (median 15 days). Prior antibiotic use due to various infections was observed in only $15(38.5 \%)$ of the patients. The crude mortality rate was $24.3 \%$ for all nosocomial infections and $53.8 \%$ for $B$. cepacia infections. B. cepacia infections were seen mostly in intensive care units. The most frequent infection was pneumonia (58.9\%). The demographic and clinical characteristics of patients are shown in table 2.

The antimicrobial susceptibility of $B$. cepacia isolates is summarized in table 3. The most active antimicrobial agents were piperacillin-tazobactam, cefoperazone-sulbactam, and carbapenems.

\section{Discussion}

Non-fermentative Gram negative rods are opportunistic pathogens responsible for nosocomial infections. Pseudomonas aeruginosa, Acinetobacter baumannii and Stenotrophomonas maltophilia are the most frequent non-fermentative agents of nosocomial infections in intensive care units. Multidrug resistance among these species can cause serious problems in the clinical setting. B. cepacia can now be added to this list because of its high transmissibility between hospitalized patients and multiple drug resistance. B. cepacia is virtually nonpathogenic in healthy hosts. It is commonly associated with colonization and pulmonary infection in $\mathrm{CF}$ patients $[1,10,11]$. However, the pathogenicity of $B$. cepacia is not always limited to individuals with CF. B. cepacia has also been recognised, albeit rarely, as causing fatal disease in healthy individuals $[12,13]$. This microorganism is associated with a wide variety of infections, including pneumonia, bacteremia, skin and soft tissue infection, genitourinary tract infection secondary to urethral instrumentation, or through exposure to contaminated solutions in hospitalized patients [4,14]. In hospitals, B. cepacia has been found to contaminate antiseptics, disinfectants, nebuliser solution, and dextrose solution [15]. Holmes et al. reported a large hospital outbreak that appeared to involve both $\mathrm{CF}$ and nonCF patients [16]. A phylogenetic analysis revealed a cross-infection by a single dominant clone of $B$. cepacia between patients with and without cystic fibrosis.

In the literature, reports of nosocomial B. cepacia infections are restricted to the epidemics [1-3,5,19]. Consistent with the nature of nosocomial epidemics, breaks in infection control as well as the high transmissibily of the microroganism are mostly responsible for the B. Cepacia epidemics. Our data do not show any epidemics, but all sporadic cases of B. cepacia infections. Though not included in the study, one can consider the rapid identification of the cases and the strict infection control measures and apply them to avoid epidemics.

The isolation and identification of B. cepacia can be difficult in the laboratory. The correct identification of this organism is important because of the high rate of cross-infection and associated virulence. Accurate identification of $B$. cepacia is difficult, and there are a few strains which are known 
Table 1. Incidence of Burkholderia cepacia infections, isolation percentages, wards, and infection sites between 2003-2007.

\begin{tabular}{|l|l|}
\hline Characteristic & Value \\
\hline No. of Burkholderia cepacia isolates & 39 \\
\hline Incidence (per 1000 admission) & 0.26 \\
\hline \% of Burkholderia cepacia among NI isolates & 0.7 \\
\hline $\begin{array}{l}\text { \% of Burkholderia cepacia } \text { among Gram } \\
\text { negative isolates } 1.0\end{array}$ & 1.0 \\
\hline Wards & No. (\%) of \\
\hline patients \\
\hline Surgical wards & $10 / 39(25.6)$ \\
\hline Intensive care units & $5 / 39(12.8)$ \\
\hline Infection site & $24 / 39(61.5)$ \\
\hline Pneumonia (included VAP) & \\
\hline Bloodstream (included catheter related BSI) & $10 / 39(25.6)$ \\
\hline Urinary tract & $2 / 39(5.1)$ \\
\hline Surgical site & $3 / 39(7.6)$ \\
\hline Skin-soft tissue & $1 / 39(2.5)$ \\
\hline NI = nosocomial infection, VAP = ventilator-associated pneumonia, BSI = Bloodstream infections
\end{tabular}

Table 2. Demographic and clinical characteristics of 39 patients with Burkholderia cepacia infection

\begin{tabular}{|l|l|}
\hline Charasteristic & Value \\
\hline Gender, male/female & $22 / 17(1.29)$ \\
\hline Crude mortality rate (\%) & 53.8 \\
\hline Age, mean+SD (year) & $54.4 \pm 23.4$ \\
\hline Setting, ICU/non-ICU & $24 / 15$ \\
\hline Duration of hospitalization (days) & $15.2 \pm 9.9$ \\
\hline Underlying diseases / risk factors & $\begin{array}{l}\text { No. (\%) of } \\
\text { patients }\end{array}$ \\
\hline Central venous catheter & $34(87.1)$ \\
\hline Urinary catheter & $30(76.9)$ \\
\hline Antiacid & $29(74.3)$ \\
\hline Mechanical ventilation & $25(64.1)$ \\
\hline Endoscopy & $22(56.4)$ \\
\hline Prior antibiotic use & $15(38.4)$ \\
\hline Tracheostomy & $14(35.8)$ \\
\hline Blood transfusion & $13(33.3)$ \\
\hline Malignancy & $11(28.2)$ \\
\hline Drainage catheter & $11(28.2)$ \\
\hline Diabetes mellitus & $6(15.3)$ \\
\hline Immunsupressive therapy & $5(12.8)$ \\
\hline Hemodialysis & $5(12.8)$ \\
\hline & \\
\hline
\end{tabular}

as the $B$. cepacia complex. The molecular taxonomic analyses placed different $B$. cepacia strains into groups known as genomovars. Although genotypic identification with molecular tests is more accurate for definitive identification, a few genomovars can be excluded according to the phenotypic characteristics [17]. As this study was a retrospective analysis and the cases were not cummulative, we could not identify genomovars of $B$. cepacia strains. Antimicrobial susceptibility tests were performed by the disk diffusion method in the study. Ideally, the susceptibility testing should have been done by the Etest or microbroth dilution method; this is a limitation for our retrospective study.

Table 3. Antimicrobial resistance rates of Burkholderia cepacia isolates (n:39)

\begin{tabular}{|l|l|}
\hline Antibiotics & Resistance, n (\%) \\
\hline Amikacin & $21(53.8)$ \\
\hline İmipenem & $18(46.1)$ \\
\hline Meropenem & $19(48.7)$ \\
\hline Netilmicin & $21(53.8)$ \\
\hline Piperacillin-tazobactam & $15(38.4)$ \\
\hline Ceftazidime & $24(61.5)$ \\
\hline Cefotaxime & $30(76.9)$ \\
\hline Cefepime & $22(56.4)$ \\
\hline Ciprofloxacin & $21(53.8)$ \\
\hline Cefoperazone-sulbactam & $17(43.5)$ \\
\hline Trimethoprim-sulfamethoxazole & $22(56.4)$ \\
\hline
\end{tabular}

The incidence of nosocomial $B$. cepacia infections was very low in our hospital $(0.26$ per 1000 admission). The ICUs (61.5\%) were the wards in which nosocomial B. cepacia infections occurred more frequently. The crude mortality was found to be $53.8 \%$ in nosocomial B. cepacia infections. The most frequent risk factors in these patients were invasive procedures such as mechanical ventilation and urinary and central venous catheterization, which were mostly related to the severity of the underlying diseases of patients in the ICU. There is no data about its incidence among nosocomial infections in surveillance studies in the literature. Most of the reports are focused on its potential to cause nosocomial outbreaks and of resistance to commonly used antibiotics. The outbreaks due to B. cepacia have frequently occurred in ICUs, and have been traced to sources related to the respiratory route, nebulised medication, mouthwash and the disinfectant $[2,3,5]$. Contaminated products, including tap, distilled, or deionized water; contaminated chlorhexidine; topical anesthetic agents; benzalkonium chloride; povidone iodine solution; and quarternary ammonium solutions were responsible for numerous outbreaks in a hospital 
setting $[1,4]$. B. cepacia has been associated with outbreaks involving infections of the bloodstream, respiratory tract, and urinary tract [4].

In our hospital, the most frequent $B$. cepacia infection was pneumonia (58.9\%), and $64.1 \%$ of the patients were under mechanical ventilation. Mechanical ventilation is a major risk factor for $B$. cepacia respiratory acquisition, and association has been described in B. cepacia outbreaks. Oropharyngeal bacterial colonization during intubation, poor cough reflex, and direct inhalation of contaminated aerosols into the lower respiratory tract have been involved in the higher risk for pneumonia of patients receiving mechanical ventilation $[2,10]$.

Bloodstream infections, including catheter related BSI, were the second most common (25.6\%) B. cepacia infection in our hospital. B. cepacia bacteremia, most often in association with polymicrobial catheter-related infection, has been reported in patients with cancer who are undergoing hemodialysis [4]. Two outbreaks of B. cepacia nosocomial infection in a neonatal intensive care unit were reported by Lee [18]. Prior long duration of an intravascular line was found to be associated with the first septicaemic episode in a case control analysis of the first outbreak. Another nosocomial outbreak of intravenous catheter-related B. cepacia bloodstream infections was reported by Nasser et al. [3].

B. cepacia is a well-known nosocomial pathogen that is intrinsically resistant to aminoglycosides and first- and second-generation cephalosporins. The multiple-antibiotic resistance of B. cepacia has been attributed to an impermeable selective outer membrane, an efflux pump mechanism, and/or the production of an inducible chromosomal betalactamase [19-21]. The antimicrobial resistance rates among B. cepacia strains were found to be high in our study. The most active antimicrobial agents against $B$. cepacia isolates were piperacillintazobactam, cefoperazone-sulbactam and carbapenems. Carbapenem resistance is reported to be as high as $48-89 \%$ among B. cepacia isolated from nosocomial infections with cystic fibrosis [22-24]. Carbapenem resistance rate in our study was approximately at the lower end of this range.

In conclusion, the incidence of nosocomial infections due to B. cepacia was rare in our hospital. No outbreak was detected during the study period. Nosocomial B.cepacia infections occured mostly in intensive care unit patients associated with invasive procedures. Its potential to cause fatal infections in the ICUs and its multidrug resistance makes its presence dangerous in hospital settings. The surveillance of $B$. cepacia infections should not be neglected, especially in the ICUs. Given the high transmissibility of the microorganism and previous epidemic reports, strict infection control measures should be applied in the case of a $B$. cepacia infection diagnosis.

\section{References}

1. Siddiqui AH, Mulligan ME, Mahenthiralingam E, Hebden J, Brewrink J, Qaiyumi S, Johnson JA, LiPuma JJ (2001) An episodic outbreak of genetically related Burkholderia cepacia among non-cystic fibrosis patients at a university hospital. Infect Control Hosp Epidemiol 22: 419-422.

2. Estivariz CF, Bhatti LI, Pati R Jensen B, Arduino MJ, Jernigan D, Lipuma JJ, Srinivasan A (2006) An outbreak of Burkholderia cepacia associated with contamination of albuterol and nasal spray. Chest 130: 1346-1353.

3. Nasser RM, Rahi AC, Haddad MF, Daoud Z, Irani-Hakime N, Almawi WY (2004) Outbreak of Burkholderia cepacia bacteremia traced to contaminated hospital water used for dilution of an alcohol skin antiseptic. Infect Control Hosp Epidemiol 25: 231-239.

4. Maschmeyer G, Göbel UB (2005) Stenotrophomonas maltophilia and Burkholderia cepacia. In: Bennett JE, Mandell GL, Dolin R, editors. Principles and Practice of Infectious Diseases. 6th ed. Philadelphia: Churchill Livingstone. 2615-2622.

5. Matrician L, Ange G, Burns S, Fanning WL, Kioski C, Cage GD, Komatsu KK (1998) Nosocomial Burkholderia cepacia infection and colonization associated with intrinsically contaminated mouthwash--Arizona, 1998. MMWR 47: 926-928.

6. Jarvis WR, Olson D, Tablan O, Martone WJ (1987) The epidemiology of nosocomial Pseudomonas cepacia infections: endemic infections. Eur J Epidemiol 3: 233236.

7. Maningo E, Watanakunakorn C (1995) Xanthomonas maltophilia and Pseudomonas cepacia in lower respiratory tracts of patients in critical care units. J Infect 31:89-92.

8. Garner JS, Jarvis WR, Emori TG, Horan TC, Hughes JM (1998) CDC definitions for nosocomial infections. Am J Infect Control 16: 128-140.

9. CLSI (Clinical and Laboratory Standards Institute). Performance Standards for Antimicrobial Susceptibility Testing; 15th Informational Supplement, M100-S15. Wayne (PA): CLSI; 2005.

10. Ramsey AH, Skonieczny P, Coolidge DT, Kurzynski TA, Proctor ME, Davis JP (2001) Burkholderia cepacia lower respiratory tract infection associated with exposure to a respiratory therapist. Infect Control Hosp Epidemiol 22: 423-426.

11. Öztürk R. (2008) Antimicrobial Treatment for Infectious Diseases with Multi-drug Resistant Pseudomonas aeruginosa, Burkholderia cepacia, and Stenotrophomonas maltophilia. ANKEM Derg 22: 36-43. [Article in Turkish]

12. Wong S, Tam AY, Yung RW, Kwan EY, Tsoi NN (1991) Pseudomonas septicaemia in apparently healthy children. Acta Paediatr Scan 80: 515 - 520.

13. Hobson R, Gould I, Govan J (1995) Burkholderia (Pseudomonas) cepacia as a cause of brain abscesses 
secondary to chronic suppurative otitis externa. Eur J Clin Microbiol Infect Dis 41: 908 - 911.

14. Aygencel G, Dizbay M, Sahin G ( 2008) Burkholderia cepacia as a cause of ecthyma gangrenosum-like lesion. Infection 36: 271-273.

15. Jones AM, Dodd ME, Webb AK (2001)Burkholderia cepacia: current clinical issues, environmental controversies and ethical dilemmas. Eur Respir J. 17: 295301.

16. Holmes A, Nolan R, Taylor R, Finley R, Riley M, Jiang RZ, Steinbach S, Goldstein R (1999) An epidemic of Burkholderia cepacia transmitted between patients with and without Cystic Fibrosis. J Infect Dis 179: 1197 - 1205.

17. Turton JF, Arif N, Hennessy D, Kaufmann ME, Pitt TL (2007) Revised approach for identification of isolates within the Burkholderia cepacia complex and description of clinical isolates not assigned to any of the known genomovars. J Clin Microbiol 45: 3105-3108.

18. Lee JKF (2008) Two outbreaks of Burkholderia cepacia nosocomial infection in a neonatal intensive care unit. Journal of Paediatrics and Child Health 44: 62-66.

19. Burns JL, Wadsworth CD, Barry JJ, Goodall CP (1996) Nucleotide sequence analysis of a gene from Burkholderia (Pseudomonas) cepacia encoding an outer membrane lipoprotein involved in multiple antibiotic resistance. Antimicrob Agents Chemother 40: 307-313.

20. Aronoff SC (1988) Outer membrane permeability in Pseudomonas cepacia: diminished porin content in a betalactam-resistant mutant and in resistant cystic fibrosis isolates. Antimicrob Agents Chemother 32: 1636-1639.

21. Trépanier S, Prince A, Huletsky A (1997) Characterization of the penA and penR genes of Burkholderia cepacia 249 which encode the chromosomal class A penicillinase and its LysR-type transcriptional regulator. Antimicrob Agents Chemother 41: 2399-2405.

22. Manno G, Ugolotti E, Belli ML, Fenu ML, Romano L, Cruciani M (2003) Use of the E test to assess synergy of antibiotic combinations against isolates of Burkholderia cepacia-complex from patients with cystic fibrosis. Eur J Clin Microbiol Infect Dis 22: 28-34.

23. Araque-Calderon Y, Miranda-Contreras L, RodriguezLemoine V, Palacios-Pru EL (2008) Antibiotic resistance patterns and SDS-PAGE protein profiles of Burkholderia cepacia complex isolates from nosocomial and environmental sources in Venezuela. Med Sci Monit 14: 49-55.

24. Bonacorsi S, Fitoussi F, Lhopital S, Bingen E (1999) Comparative in vitro activities of meropenem, imipenem, temocillin, piperacillin, and ceftazidime in combination with tobramycin, rifampin, or ciprofloxacin against Burkholderia cepacia isolates from patients with cystic fibrosis. Antimicrob Agents Chemother 43: 213-217.

\section{Corresponding author \\ Dr. Ozlem Guzel Tunccan \\ oguzel@gazi.edu.tr}

Conflict of interest: No conflict of interest is declared. 